\title{
Electron Localization Involving Doubly Excited States in Broadband Extreme Ultraviolet Ionization of $\mathbf{H}_{2}$
}

\author{
Andreas Fischer, ${ }^{1 *}$ Alexander Sperl, ${ }^{1}$ Philipp Cörlin, ${ }^{1}$ Michael Schönwald, ${ }^{1}$ Helga Rietz, ${ }^{1}$ Alicia Palacios, ${ }^{2}$ \\ Alberto González-Castrillo, ${ }^{2}$ Fernando Martín, ${ }^{2,3}$ Thomas Pfeifer, ${ }^{1}$ Joachim Ullrich, ${ }^{1,4}$ \\ Arne Senftleben, ${ }^{1}$ and Robert Moshammer ${ }^{1, \dagger}$ \\ ${ }^{1}$ Max-Planck-Institut für Kernphysik, Saupfercheckweg 1, 69117 Heidelberg, Germany \\ ${ }^{2}$ Departamento de Química, Módulo 13, Universidad Autónoma de Madrid, 28049 Madrid, Spain \\ ${ }^{3}$ Instituto Madrileño de Estudios Avanzados en Nanociencia (IMDEA-Nanociencia), Cantoblanco, 28049 Madrid, Spain \\ ${ }^{4}$ Physikalisch-Technische Bundesanstalt, Bundesallee 100, 38116 Braunschweig, Germany
}

(Received 11 March 2013; published 20 May 2013)

\begin{abstract}
Dissociative single ionization of $\mathrm{H}_{2}$ induced by extreme ultraviolet photons from an attosecond pulse train has been studied in a kinematically complete experiment. Depending on the electron kinetic energy and the alignment of the molecule with respect to the laser polarization axis, we observe pronounced asymmetries in the relative emission directions of the photoelectron and the $\mathrm{H}^{+}$ion. The energydependent asymmetry pattern is explained by a semiclassical model and further validated by fully quantum mechanical calculations, both in very good agreement with the experiment.
\end{abstract}

DOI: 10.1103/PhysRevLett.110.213002

PACS numbers: 33.80.Eh

Molecular hydrogen is the simplest molecule that exhibits electron correlation and doubly excited states. Having a total energy above the ionization threshold, they are embedded in the single-ionization continuum and thus autoionize via emission of an electron on a time scale that is comparable to the dissociation time. The dynamical interplay between the electronic and nuclear motion in autoionization has been the subject of research $[1,2]$. It has raised particular attention with the advent of ultrashort light pulses whose durations may permit real-time imaging and control of these processes [3].

Theoretical investigations suggested that the symmetry of hydrogenic molecules $\left(\mathrm{H}_{2}, \mathrm{D}_{2}, \mathrm{HD}\right)$ can be broken after light-induced ionization [4-6]. This is a consequence of interferences arising from a coherent superposition of ionic molecular states with different parities resulting in the localization of the remaining bound electron. Several experiments demonstrated this for multiphoton processes [3,7-10] as well as for single-photon transitions [6,11,12]. However, a fully differential analysis of the asymmetry arising from electron localization in photoionization induced by spectrally broad laser pulses has never been reported.

Here, we present experimental data on $\mathrm{H}_{2}$ photoionization using an attosecond pulse train. Using a semiclassical model [13-15] based on the WKB approximation, we demonstrate that both the origin of the observed asymmetry and its oscillations as a function of the kinetic energies of the charged fragments can be accounted for fully. The present findings are further confirmed by a fully quantum mechanical calculation.

In the experiment $(35 \pm 10)$ fs IR pulses, provided by a commercial laser system, are spectrally broadened in a hollow core fiber and recompressed to $(15 \pm 5)$ fs using chirped mirrors. Using high harmonic generation [16] in argon followed by an aluminum filter to remove the fundamental light, we reach extreme ultraviolet (XUV) photon energies between 16 and $37 \mathrm{eV}$. The high harmonics are emitted as attosecond pulse trains where the number of pulses depends on the duration of the generating laser pulse and is here estimated to be less than 10. The XUV beam is then focused into a supersonic jet of hydrogen gas. The focal spot is placed in the center of a reaction microscope [17] enabling the measurement of the individual momenta of all charged particles emerging from the ionization reaction allowing us to completely characterize the kinematics. Moreover, it allows the identification of ion species via their mass-to-charge ratio and consequently to distinguish between possible ionization channels. In $\mathrm{H}_{2}$, these are the dissociative ionization and dominantly the production of stable molecular $\mathrm{H}_{2}^{+}$ions.

Here, we concentrate on the dissociative ionization channel

$$
\mathrm{H}_{2} \stackrel{\mathrm{XUV}}{\rightarrow} \mathrm{H}+\mathrm{H}^{+}+e^{-},
$$

which has significant contributions from autoionization of doubly excited states. The momentum of the neutral $\mathrm{H}$ atom cannot be measured directly, but it can be deduced from conservation of momentum. For further analysis we introduce the kinetic energy release (KER) as the sum of the proton and the neutral hydrogen energy.

Figure 1 shows the measured energy correlation between KER and the electron kinetic energy $E_{e}$ for events where the proton momentum vector deviates less than $35^{\circ}$ from the polarization axis. We will refer to this situation as parallel transitions. Assuming single-photon absorption, events involving the same photon energy $E_{\gamma}$ need to appear in Fig. 1 on diagonals given by 


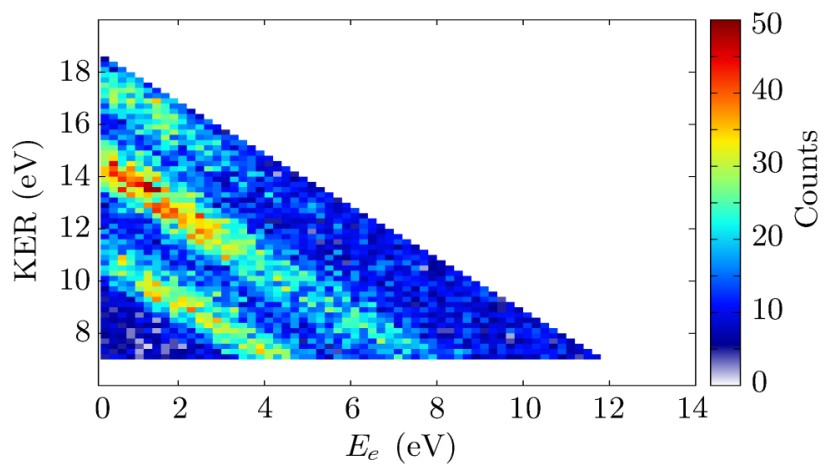

FIG. 1 (color online). Distribution of nuclear kinetic energy release (KER) versus electron kinetic energy $\left(E_{e}\right)$ measured for dissociative photoionization of $\mathrm{H}_{2}$ aligned parallel to the polarization at photon energies between 25 and $37 \mathrm{eV}$.

$$
\mathrm{KER}+E_{e}+E_{\text {limit }}=E_{\gamma}
$$

where $E_{\text {limit }}$ is the potential energy of the final system for infinite internuclear separation. For $\mathrm{H}_{2}^{+}$, the two lowest values for $E_{\text {limit }}$ are 18.08 and $28.28 \mathrm{eV}$ with respect to the $\mathrm{H}_{2}$ ground state. The first value corresponds to the limit of the two lowest states $X$ and $A$ (see Fig. 2) of the $\mathrm{H}_{2}^{+}$ion. Only these states contribute significantly to our data, since dissociation to the second limit would create additional lines in the energy correlation map (Fig. 1) at $10.2 \mathrm{eV}$ less energy which are not present in the data. Therefore, setting $E_{\text {limit }}=$ $18.08 \mathrm{eV}$ in Eq. (2) and considering a photon energy of $1.7 \mathrm{eV}$ for the fundamental laser beam, the three prominent lines in Fig. 1 with KER $+E_{e} \approx 10.8,14.2$, and $17.6 \mathrm{eV}$ are assigned to the harmonic orders HH17, HH19, and HH21, respectively. These allow for direct dissociative ionization via the $A^{2} \Sigma_{u}^{+}$state and the excitation of the doubly excited $Q_{1}$ states, as can be seen in the potential energy curves of Fig. 2.

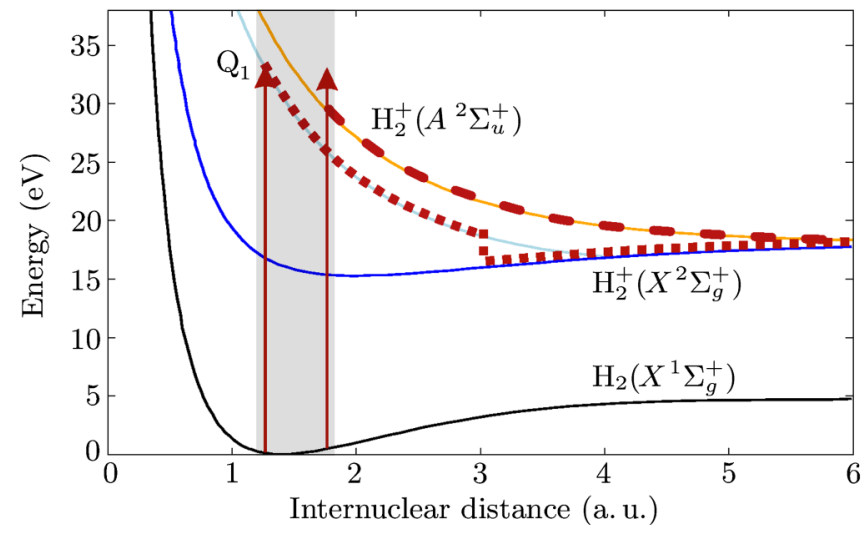

FIG. 2 (color online). Potential energy curves for the relevant $\mathrm{H}_{2}$ and $\mathrm{H}_{2}^{+}$states. Two possible dissociation pathways yielding the same KER and electron energy are indicated: direct ionization to the dissociative $A^{2} \Sigma_{u}^{+}$state of the $\mathrm{H}_{2}^{+}$(dashed line) and excitation of doubly excited states of the $\mathrm{H}_{2}\left(Q_{1}{ }^{1} \Sigma_{u}^{+}\right)$manifold followed by dissociation and subsequent autoionization to the $\mathrm{H}_{2}^{+}\left(X^{2} \Sigma_{g}^{+}\right)$state (dotted line). Figure adapted from [6].
Even though in Fig. 2 only one $Q_{1}$ state is depicted, there are bands of doubly excited states, each converging to a one-electron continuum. In the limit of high excitations the $Q_{1}$ states approach the $\mathrm{H}_{2}^{+}\left(A^{2} \Sigma_{u}^{+}\right)$state of the molecular ion. All $Q_{1}$ states are antibinding and lead to the dissociation of the molecule.

Using dipole selection rules and symmetry arguments, we identify the states contributing to the asymmetry effect (Fig. 2). The point group of molecular hydrogen is $D_{\infty h}$, where the dipole operator for parallel transitions belongs to the irreducible representation $\Sigma_{u}^{+}$. As the ground state of $\mathrm{H}_{2}$ is of $\Sigma_{g}^{+}$symmetry, only $Q_{1}$ states of $\Sigma_{u}^{+}$symmetry are excited by one-photon transitions, while ionization can in principle leave the molecular ion in any symmetry. However, considering the available states converging to the lowest dissociation limit, we only populate the $\mathrm{H}_{2}^{+}\left(X^{2} \Sigma_{g}^{+}\right)$ and the $\mathrm{H}_{2}^{+}\left(A^{2} \Sigma_{u}^{+}\right)$states in the ionization process.

We are now investigating vector correlations between the observed fragments. Therefore, we define a molecularframe asymmetry $A$ using the angle $\alpha$ between the momentum vectors of the proton and the electron

$$
A=\frac{N_{<90^{\circ}}-N_{>90^{\circ}}}{N_{<90^{\circ}}+N_{>90^{\circ}}},
$$

with the number $N_{>90^{\circ}}$ of events where $\alpha$ is greater than $90^{\circ}$ and the number $N_{<90^{\circ}}$ of events where $\alpha$ is less than $90^{\circ}$. In other words, $A$ is a measure for the asymmetry of the molecular-frame electron angular distribution with respect to the (arbitrarily oriented) proton momentum vector. Measured electron angular distributions for positive and negative symmetry are shown in the inset of Fig. 3(a) together with the corresponding orientation of the molecular ion. In Fig. 3(a) the asymmetry is plotted in the energy correlation map revealing oscillating structures. The oscillation is mainly a function of $E_{e}$, whereas the KER has much smaller influence on the asymmetry. Figure 3 also reveals that a projection of the data onto the KER axis, as well as a projection onto the electron energy axis, may lead to decreasing asymmetry when the spectral profile of the photons is broad $[18,19]$.

Asymmetry, as defined here, results from the interference of two molecular states with opposite parity yielding the same observables after dissociating along their individual pathways. This means in this context that both pathways (dashed and dotted lines in Fig. 2) have to yield the same kinetic energy of the electron $E_{e}$ and the same KER. In the present case, the two ionic states converging to the first dissociation limit have opposite parity. From Fig. 2 we can see that the direct population of the two ionic states from the ground state with the same photon energy cannot lead to any asymmetry because both the kinetic energies of the photoelectron and the KER will be very different. But the situation changes with the inclusion of the doubly excited $Q_{1}{ }^{1} \Sigma_{u}^{+}$states. Because these states have autoionization times of a few femtoseconds, which are comparable 

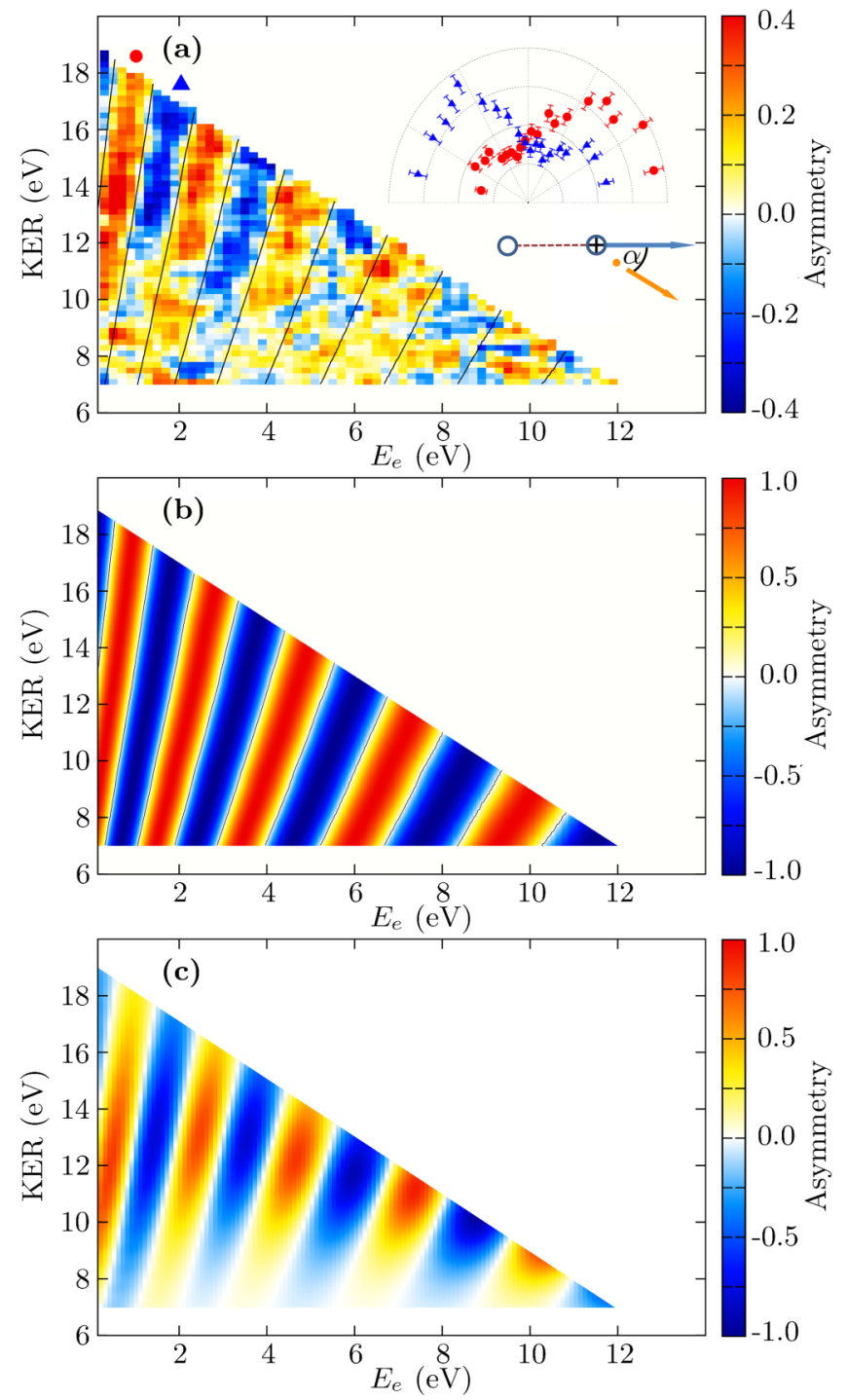

FIG. 3 (color online). Asymmetry for electron emission in dissociative photoionization of $\mathrm{H}_{2}$. (a) Measured asymmetry and (b) results of the semiclassical simulation. The black contours are lines of constant asymmetry $A=0$ and copied to the experimental plot for comparison. The inset in (a) features the distribution of the electron-proton angle $\alpha$ for positive (circles) and negative (triangles) values of $A$. (c) Fully quantum mechanical calculation.

to the dissociation time of the molecule, the energy is distributed between the nuclei and the ejected electron.

In the following we introduce a semiclassical model, taking quantum-interference effects into account, describing the dissociation along two pathways (dashed and dotted lines). The first pathway (dashed line) describes the direct ionization into the $\mathrm{H}_{2}^{+}\left(A^{2} \Sigma_{u}^{+}\right)$state which leads to dissociation of the molecule. In the second case the neutral $\mathrm{H}_{2}$ is doubly excited to one of the $\mathrm{H}_{2}\left(Q_{1}^{1} \Sigma_{u}^{+}\right)$states. Since the state is antibinding, the nuclei start to move apart. At a certain internuclear distance the molecule may autoionize to the $\mathrm{H}_{2}^{+}\left(X^{2} \Sigma_{g}^{+}\right)$state, ejecting an electron with an energy corresponding to the energy difference of both states. Interference occurs if the KER and the electron energy are the same for both pathways.

The localization of the emitted electron $e$ and the proton $p$ can be described in a "left-right" basis defined by

$$
\begin{aligned}
& \left|p_{\rightarrow}\right\rangle=\left|X^{2} \Sigma_{g}^{+}\right\rangle-\left|A^{2} \Sigma_{u}^{+}\right\rangle, \quad\left|p_{\leftarrow}\right\rangle=\left|X^{2} \Sigma_{g}^{+}\right\rangle+\left|A^{2} \Sigma_{u}^{+}\right\rangle, \\
& \left|e_{\rightarrow}\right\rangle=\left(\left|\sigma_{g}\right\rangle+\left|\sigma_{u}\right\rangle\right), \quad\left|e_{\leftarrow}\right\rangle=\left(\left|\sigma_{g}\right\rangle-\left|\sigma_{u}\right\rangle\right),
\end{aligned}
$$

where $\left|X^{2} \Sigma_{g}^{+}\right\rangle$and $\left|A^{2} \Sigma_{u}^{+}\right\rangle$describe the wave function of the bound electron and $\left|\sigma_{g}\right\rangle$ and $\left|\sigma_{u}\right\rangle$ the wave function of the emitted electron. With those definitions, a basis describing co- and counterpropagation of the entangled ion and the electron, as needed to describe the observed asymmetry, is constructed:

$\begin{array}{llrl}\left|\phi_{\rightleftarrows}\right\rangle=\left|p_{\rightarrow}\right\rangle \otimes\left|e_{\leftarrow}\right\rangle, & & \left|\phi_{\leftrightarrows}\right\rangle=\left|p_{\leftarrow}\right\rangle \otimes\left|e_{\rightarrow}\right\rangle, \\ \left|\phi_{\rightrightarrows}\right\rangle=\left|p_{\rightarrow}\right\rangle \otimes\left|e_{\rightarrow}\right\rangle, & & \left|\phi_{\sqsubseteq}\right\rangle=\left|p_{\leftarrow}\right\rangle \otimes\left|e_{\leftarrow}\right\rangle .\end{array}$

In both basis sets normalization and antisymmetrization are neglected.

Furthermore, we need to describe our final state at the detector. In the experiment the detectors are located far away from the reaction volume; therefore, the contributing doubly excited states will all have autoionized. The final state can be written as

$$
|\Psi\rangle=c_{1}\left|X^{2} \Sigma_{g}\right\rangle \otimes\left|\sigma_{u}\right\rangle+c_{2}\left|A^{2} \Sigma_{u}\right\rangle \otimes\left|\sigma_{g}\right\rangle,
$$

where $\left|\sigma_{g}\right\rangle$ is the photoelectron from the direct ionization into the $\mathrm{H}_{2}^{+}\left(A^{2} \Sigma_{u}^{+}\right)$state and $\left|\sigma_{u}\right\rangle$ describes the state of the electron that is ejected when the doubly excited state autoionizes. It must be emphasized that, by reducing the final state to the coherent sum of only two amplitudes in Eq. (6), we have made two important assumptions: (i) only two partial waves for the ejected electron contribute to the angular distribution, i.e., the lowest angular momentum ( $l=0$ associated to the $A^{2} \Sigma_{u}^{+}$state and $l=1$ associated to the $X^{2} \Sigma_{g}^{+}$), and (ii) the $X^{2} \Sigma_{g}^{+}$state can only be populated by autoionization from one of the doubly excited states. These are reasonable approximations in the photon energy range considered in this work. To get the probabilities $n_{\leftrightarrows}, n_{\rightleftarrows}, n_{\rightleftarrows}$, and $n_{\rightrightarrows}$ that the system will end up in either of the four states defined in Eq. (5), we project the final-state wave function $|\Psi\rangle$ onto them.

With these probabilities one can define the asymmetry as

$$
a=\frac{\left(n_{\rightrightarrows}+n_{\rightleftarrows}\right)-\left(n_{\rightleftarrows}+n_{\leftrightarrows}\right)}{\left(n_{\rightrightarrows}+n_{\Xi}\right)+\left(n_{\rightleftarrows}+n_{\leftrightarrows}\right)} .
$$

In the limit of many events, with $n_{\rightleftarrows}$ and $n_{\leftrightarrows}$ contributing to $N_{>90^{\circ}}$ and $n_{\rightrightarrows}$ and $n_{\leftleftarrows}$ to $N_{<90^{\circ}}$, Eq. (7) converges to the previously defined asymmetry $A$ of Eq. (3). Carrying out the projection, the asymmetry $a$ defined by Eq. (7) can be written as 


$$
a=-\frac{2 \operatorname{Re}\left[c_{1}^{*} c_{2}\right]}{\left|c_{1}\right|^{2}+\left|c_{2}\right|^{2}}
$$

Since Eq. (8) depends on only two coefficients, this expression can readily be used to qualitatively compute the asymmetry [Fig. 3(b)].

In order to simulate the asymmetry [Eq. (8)], we need to determine the coefficients $c_{1}$ and $c_{2}$. Both complex coefficients may be written in their polar form

$$
c_{1}=r_{1} e^{i \chi_{1}}, \quad c_{2}=r_{2} e^{i \chi_{2}},
$$

where $r_{1,2}$ describes the amplitudes of the occupation of the states and $\chi_{1,2}$ their phases. Because $r_{1,2}$ determine only the magnitude of the asymmetry [see Eq. (8)], which is not under investigation here, and can only be obtained by a full quantum mechanical calculation, we assume them to be unity. The phases, however, can be split into two parts, $\chi_{1,2}=$ $\varphi_{1,2}+\xi_{1,2}$, where $\xi_{1,2}$ are the phases acquired in the transitions between electronic states and $\varphi_{1,2}$ are the dynamical nuclear phases. The $\xi_{1,2}$ are accessible by the full quantum calculation or by comparing spectra obtained from different isotopes [20]. However, the dynamical phases $\varphi_{1,2}$ can be modeled by our semiclassical model. To compute them we have to find the dissociation pathways for each set of photon energy, KER, and electron energy. One possible set of pathways is depicted in Fig. 2. Once we know the two pathways we can calculate the phases. In WKB approximation, we get

$$
\varphi_{1,2}=\int_{R_{1,2}^{t}}^{\infty} p_{1,2}(R) d R
$$

where $R_{1,2}^{\mathrm{t}}$ are the internuclear distances at which the XUV excitation occurs and $p_{1,2}(R)$ the nuclear momenta. It is assumed that the initial kinetic energy right after the excitation is zero. Further, we assume that the kinetic energy at the point of the autoionization (dotted pathway) is conserved. The phases of these two integrals are then used together with Eq. (8) to compute the asymmetry.

The result of the simulation [Fig. 3(b)], where we set $\xi_{1}-\xi_{2}=\pi$, is in very good agreement with the measurement [Fig. 3(a)]. The value $\xi_{1}-\xi_{2}=\pi$ is close to that obtained by averaging the phase difference resulting from the $a b$ initio calculation in the Franck-Condon region and in the photon energy range considered in this work. The black contour lines of symmetric ejection $a=0$ are calculated by the simulation and overlaid on both energy correlation maps to enable better comparison with the measurement.

All phases are inherently accounted for in a fully quantum mechanical treatment of the problems, which we have performed using a previously described method [21]. The resulting asymmetry, displayed in Fig. 3(c), yields the same oscillations as the semiclassical simulation while simultaneously reproducing the fading contrast at smaller KER seen in the experiment.

In the case of perpendicular transitions, which we select by choosing events with an angle of more than $80^{\circ}$ between the proton emission direction and the polarization

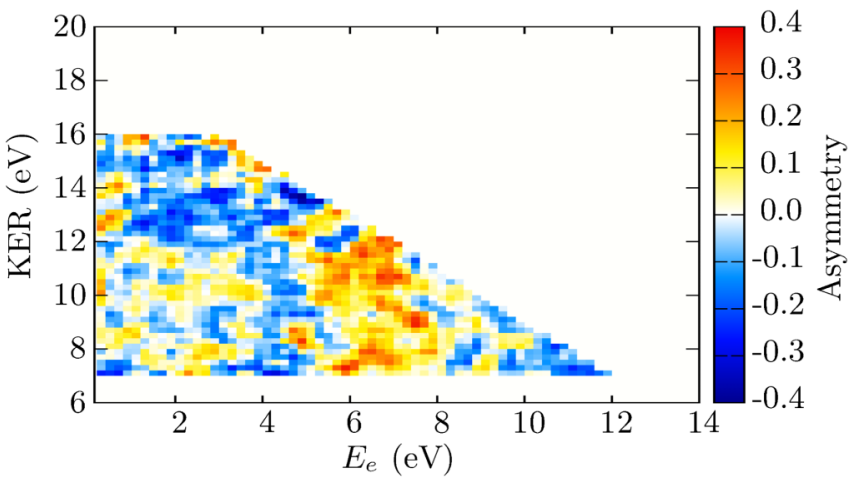

FIG. 4 (color online). Asymmetry in the perpendicular case. Also note that there is an additional energy cutoff at $16 \mathrm{ev}$ due to a lack of statistics in this region.

axis, the dipole selection rule allows only the excitation of doubly excited states of $\Pi_{u}$ symmetry. Among these, the $Q_{1}^{1} \Pi_{u}$ states have a very long lifetime and therefore do not efficiently contribute to the ionization. We therefore expect no asymmetry. Figure 4, showing the experimental results, however, still exhibits a significant asymmetry, which can be explained by a different process taking the doubly excited $Q_{2}$ states into consideration as previously observed and described by [6].

In conclusion, we measured and analyzed the energydependent electron-proton asymmetry, caused by the interference of the $\mathrm{H}_{2}^{+}\left(X^{2} \Sigma_{g}^{+}\right)$and the $\mathrm{H}_{2}^{+}\left(A^{2} \Sigma_{u}^{+}\right)$state in the dissociation process. We found very good agreement between the measurement and our semiclassical simulation that fully accounts for the period of the oscillation and does so with very high accuracy. Since it does not model the transition from the $\mathrm{H}_{2}$ ground state to the $\mathrm{H}_{2}\left(Q_{1}^{1} \Sigma_{u}^{+}\right)$and the $\mathrm{H}_{2}^{+}\left(A^{2} \Sigma_{u}^{+}\right)$ionic state nor does it consider the decay of the $Q_{1}$ state to the $\mathrm{H}_{2}^{+}\left(X^{2} \Sigma_{g}^{+}\right)$state, it cannot describe the amplitude of the asymmetry oscillation, which is successfully done by our fully quantum mechanical treatment. Additionally, we have explained why this asymmetry is much less pronounced in the case of the perpendicular transitions which further confirms our analysis. Because, in contrast to synchrotron experiments, we used attosecond pulse trains which lead to broadband excitations, this experiment paves the road for future pump-probe experiments with control over the dissociative ionization of molecules [22].

We thank K. Simeonidis for his great contribution in setting up the experiment and B. Feuerstein for fruitful discussions. A. G.-C., A. P., and F. M. acknowledge allocation of computer time by CCC-UAM and BSC Mare Nostrum and financial support from the Advanced Grant of the European Research Council XCHEM 290853, the European grants MC-RG ATTOTREND and ITN CORINF, the European COST Actions CM0702 (CUSPFEL) and CM1204 (XLIC), the MICINN projects No. FIS2010-15127 and No. CSD 2007-00010, and the ERA-Chemistry project No. PIM2010EEC-00751. 
*andreas.fischer@mpi-hd.mpg.de

†robert.moshammer@mpi-hd.mpg.de

[1] M. Iwai, S. Lee, and H. Nakamura, Phys. Rev. A 47, 2686 (1993).

[2] J.R. Machacek, V.M. Andrianarijaona, J.E. Furst, A. L. D. Kilcoyne, A. L. Landers, E.T. Litaker, K.W. McLaughlin, and T. J. Gay, J. Phys. B 44, 045201 (2011).

[3] G. Sansone, F. Kelkensberg, J. F. Pérez-Torres, F. Morales, M. F. Kling, W. Siu, O. Ghafur, P. Johnsson, M. Swoboda, E. Benedetti, F. Ferrari, F. Lépine, J. L. Sanz-Vicario, S. Zherebtsov, I. Znakovskaya, A. L'Huillier, M. Y. Ivanov, M. Nisoli, F. Martín, and M. J. J. Vrakking, Nature (London) 465, 763 (2010).

[4] E. Charron, A. Giusti-Suzor, and F. H. Mies, Phys. Rev. Lett. 75, 2815 (1995).

[5] B. Sheehy, B. Walker, and L. F. DiMauro, Phys. Rev. Lett. 74, 4799 (1995).

[6] F. Martín, J. Fernández, T. Havermeier, L. Foucar, T. Weber, K. Kreidi, M. Schöffler, L. Schmidt, T. Jahnke, O. Jagutzki, A. Czasch, E. P. Benis, T. Osipov, A. L. Landers, A. Belkacem, M. H. Prior, H. Schmidt-Böcking, C. L. Cocke, and R. Dörner, Science 315, 629 (2007).

[7] M. F. Kling, C. Siedschlag, A. J. Verhoef, J. I. Khan, M. Schultze, T. Uphues, Y. Ni, M. Uiberacker, M. Drescher, F. Krausz, and M. J. J. Vrakking, Science 312, 246 (2006).

[8] M. Kremer, B. Fischer, B. Feuerstein, V. L. B. de Jesus, V. Sharma, C. Hofrichter, A. Rudenko, U. Thumm, C. D. Schröter, R. Moshammer, and J. Ullrich, Phys. Rev. Lett. 103, 213003 (2009).

[9] K. P. Singh, F. He, P. Ranitovic, W. Cao, S. De, D. Ray, S. Chen, U. Thumm, A. Becker, M. M. Murnane, H.C. Kapteyn, I. V. Litvinyuk, and C. L. Cocke, Phys. Rev. Lett. 104, 023001 (2010).
[10] F. Kelkensberg, W. Siu, J. F. Pérez-Torres, F. Morales, G. Gademann, A. Rouzée, P. Johnsson, M. Lucchini, F. Calegari, J. L. Sanz-Vicario, F. Martín, and M. J. J. Vrakking, Phys. Rev. Lett. 107, 043002 (2011).

[11] A. Lafosse, M. Lebech, J.C. Brenot, P. M. Guyon, L. Spielberger, O. Jagutzki, J. C. Houver, and D. Dowek, J. Phys. B 36, 4683 (2003).

[12] P. Billaud, M. Géléoc, Y. J. Picard, K. Veyrinas, J. F. Hergott, S. Marggi Poullain, P. Breger, T. Ruchon, M. Roulliay, F. Delmotte, F. Lepetit, A. Huetz, B. Carré, and D. Dowek, J. Phys. B 45, 194013 (2012).

[13] W. H. Miller, J. Chem. Phys. 52, 3563 (1970).

[14] C. J. Latimer, K. F. Dunn, N. Kouchi, M. A. McDonald, V. Srigengan, and J. Geddes, J. Phys. B 26, L595 (1993).

[15] T. J. Reddish, A. Padmanabhan, M. A. MacDonald, L. Zuin, J. Fernández, A. Palacios, and F. Martín, Phys. Rev. Lett. 108, 023004 (2012).

[16] P. Agostini and L. F. DiMauro, Rep. Prog. Phys. 67, 813 (2004).

[17] J. Ullrich, R. Moshammer, A. Dorn, R. Dörner, L. P. H. Schmidt, and H. Schmidt-Böcking, Rep. Prog. Phys. 66, 1463 (2003).

[18] J. F. Pérez-Torres, F. Morales, J. L. Sanz-Vicario, and F. Martín, Phys. Rev. A 80, 011402 (2009).

[19] A. González-Castrillo, A. Palacios, F. Catoire, H. Bachau, and F. Martín, J. Phys. Chem. A 116, 2704 (2012).

[20] A. Palacios, J. Feist, A. González-Castrillo, J. L. SanzVicario, and F. Martín, ChemPhysChem 14, 1456 (2013).

[21] A. Palacios, H. Bachau, and F. Martín, Phys. Rev. A 75, 013408 (2007).

[22] R. E. F. Silva, P. Rivière, and F. Martín, Phys. Rev. A 85, 063414 (2012). 